\title{
Fluxon modes in stacked Josephson junctions: The role of linear modes
}

\author{
Madsen, Søren Peder; Pedersen, Niels Falsig
}

Published in:

Physical Review B Condensed Matter

Link to article, DOI:

10.1103/PhysRevB.69.064507

Publication date:

2004

Document Version

Publisher's PDF, also known as Version of record

Link back to DTU Orbit

Citation (APA):

Madsen, S. P., \& Pedersen, N. F. (2004). Fluxon modes in stacked Josephson junctions: The role of linear modes. Physical Review B Condensed Matter, 69(6), 064507. https://doi.org/10.1103/PhysRevB.69.064507

\section{General rights}

Copyright and moral rights for the publications made accessible in the public portal are retained by the authors and/or other copyright owners and it is a condition of accessing publications that users recognise and abide by the legal requirements associated with these rights.

- Users may download and print one copy of any publication from the public portal for the purpose of private study or research.

- You may not further distribute the material or use it for any profit-making activity or commercial gain

- You may freely distribute the URL identifying the publication in the public portal 


\title{
Fluxon modes in stacked Josephson junctions: The role of linear modes
}

\author{
S. Madsen* and N. F. Pedersen ${ }^{\dagger}$ \\ Oersted-DTU, Section of Electric Power Engineering, Technical University of Denmark, DK-2800 Kgs. Lyngby, Denmark
}

(Received 2 June 2003; revised manuscript received 29 July 2003; published 20 February 2004)

\begin{abstract}
Plasma modes in stacked Josephson junctions are easily understood analytically from a linearization of the coupled sine-Gordon equation describing the system. We demonstrate here by numerical methods that the analytically derived symmetries of the plasma modes are carried over to the fluxon modes. Using this fact we are, with a few exceptions, able to predict and construct a full family of Josephson fluxon modes without using numerical methods. The nature of the locking mechanism needed to create the technologically important in-phase fluxon modes is discussed.
\end{abstract}

DOI: 10.1103/PhysRevB.69.064507

PACS number(s): 74.50.+r, 05.45.Yv, 85.25.Cp

\section{INTRODUCTION}

Fluxon dynamics in high-temperature cuprate superconductors is a topic of considerable interest in recent years. One of the motivations has been the potential for applications both for high-frequency electronics and for large-scale devices, and here the BSCCO family of superconductors has been particularly investigated. ${ }^{1-7}$ In both cases the fluxon dynamics of the Josephson fluxons are particularly important to understand. As examples we mention that for electronic applications understanding the mechanisms that lead to inphase fluxon motion in the different layers is essential, and for power applications it is of utmost interest to understand why the magnetic flux is pinned only at lower temperatures in BSCCO material.

The dynamics of Josephson fluxons in a stack of $N$ copper-oxide planes are governed by a system of $N$ coupled sine-Gordon equations that are extremely nonlinear and, apart from the simplest cases, only understandable by numerical methods. For initial conditions with at most one fluxon in each layer, we demonstrate below that a family of fluxon modes can be easily predicted from the symmetries of the plasma modes, which are easily obtained analytically from a linearization of the system of $N$ coupled sine-Gordon equations. A few exceptions to the predictions exist and will be discussed separately.

We note that for BSCCO material, well-defined samples can be made and microwave measurements on both plasma and fluxon excitations can be done. ${ }^{8}$

The paper is organized in the following way. In Sec. II we introduce the inductively coupled Josephson-junction model, leading to the coupled sine-Gordon system. The plasma modes are derived analytically in Sec. III. In Sec. IV we attempt to understand analytically for the simplest nontrivial case, $N=3$, the nature of the locking mechanism leading to in-phase motion of the fluxons in the different layers. In Sec. $\mathrm{V}$ we show by numerical simulations that $N$ fluxon modes with at most one fluxon in each layer can be predicted by the symmetries of the $N$ plasma modes obtained analytically. Finally a summary with the most important results concludes the paper.

\section{THEORY}

The geometry of the system under consideration is sketched in Fig. 1.
Assuming identical parameters for the layers, the equations for a stack with $N+1$ superconducting layers and $N$ insulating layers can be written in the compact form ${ }^{9}$

$$
\mathbf{J}=\mathbf{S}^{-1} \boldsymbol{\varphi}_{x x},
$$

where the $i$ th element of $\boldsymbol{\varphi}, \varphi^{i}$, is the gauge-invariant phase difference across insulating layer $i$, and the $N \times N$ coupling matrix $\mathbf{S}$ has the following form:

$$
\mathbf{S}=\left(\begin{array}{ccccc}
1 & S & & & \\
S & 1 & S & & \\
& S & 1 & S & \\
& & \ddots & \ddots & \ddots
\end{array}\right),
$$

with $S$ being the coupling parameter between the layers. The vector $\mathbf{J}$ is the current in the $z$ direction, and has the following components:

$$
J^{i}=\varphi_{t t}^{i}+\alpha \varphi_{t}^{i}+\sin \varphi^{i}-\gamma^{i}
$$

where the $\alpha$ term is a dissipative term, and $\gamma^{i}$ is the biacurrent in the $i$ th junction in the $z$ direction. We will in this paper only consider the case where $\gamma^{i}= \pm \gamma^{j}$.

Here Eqs. (1) - (3) have been written in appropriately normalized units. Space $x$ is normalized to the Josephson penetration depth $\lambda_{J}=\sqrt{\hbar / 2 e \mu_{0} J^{c} d^{\prime}}$ and time $t$ is normalized to the inverse plasma frequency $\omega_{0}^{-1}=\sqrt{\hbar C / 2 e J^{c}}$, where the symbols have their usual meaning. Using these units the con-

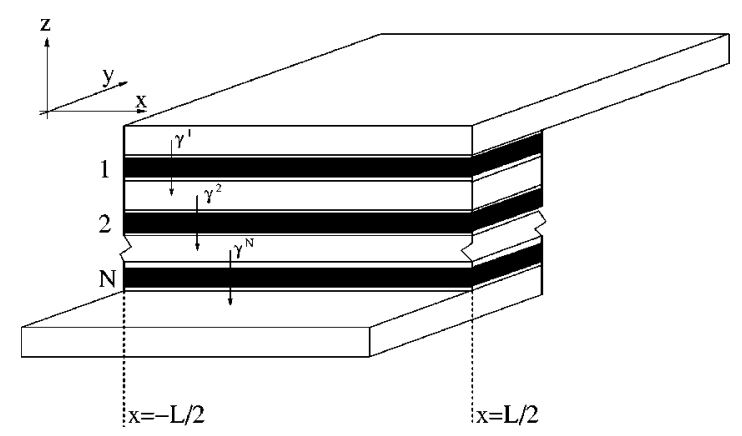

FIG. 1. The geometry of the stacked junctions. Black layers are insulators. 
stants for damping, $\alpha$, bias current, $\gamma^{i}$, and layer-to-layer coupling, $S$, all come out in dimensionless units. See Ref. 10 for details.

It is well known that the system can be solved analytically for the simplest case $\alpha=\gamma^{i}=S=0$; a particularly important solution is the familiar $2 \pi$ kink, often referred to as a soliton:

$$
\varphi^{i}(x, t)=4 \tan ^{-1} \exp \left(\sigma^{i} \frac{x-v^{i} t-x_{0}^{i}}{\sqrt{1-\left(v^{i}\right)^{2}}}\right),
$$

where $\sigma^{i}= \pm 1$ and $(+)$ corresponds to a soliton and ( - ) to an antisoliton. $v^{i}$ is the soliton velocity and $x_{0}^{i}$ is the position at $t=0$. It is worth noting that for both low $-T_{c}$ artificial Josephson stacks and for high- $T_{c}$ BSCCO-like systems realistic values of the three parameters are small, ${ }^{9}$ i.e., $0 \leqslant \alpha$ $\leqslant 0.2,0 \leqslant\left|\gamma^{i}\right| \leqslant 1$, and $-0.5<S \leqslant 0$. Due to this fact, perturbation theory often works well for these systems. ${ }^{11,12}$ For the superconducting systems discussed here, a soliton is a (Josephson) fluxon containing one quantum of magnetic flux. Correspondingly, an antisoliton corresponds to an antifluxon.

To completely define the dynamical state of the system, Eqs. (1) should be supplemented with boundary conditions. The boundary conditions depend on the geometry of the system; the geometry depicted in Fig. 1 is described by the following boundary conditions:

$$
\varphi_{x}^{i}(-L / 2, t)=\varphi_{x}^{i}(L / 2, t)=0, \quad i=1, \ldots, N .
$$

These boundary conditions are often referred to as reflective, because when a fluxon collides with the boundary, it can be reflected as an antifluxon. ${ }^{13}$

Other types of boundary conditions are also possible. An important example is the annular geometry giving rise to periodic boundary conditions. These are frequently used in numerical simulations, because they simulate somewhat a Josephson junction of infinite length. In this work we always use the reflective boundary conditions in our numerical simulations, but assume infinite length in the analytical calculations. It should also be noted that the annular geometry is also of experimental interest. ${ }^{14,15}$

\section{THE PLASMA MODES}

The plasma modes, or the small-signal limit, of the system (1) have been derived previously in Refs. 5 and 16-19. We will here quickly review the derivation. Let us start by assuming that we have no loss term, i.e., $\alpha=0$, and that $\varphi^{i}$ can be written in the following form:

$$
\varphi^{i}=a^{i} \cos (k x-\omega t)+\sin ^{-1} \gamma^{i} .
$$

Assuming that the amplitudes $a^{i} \ll 1$, i.e., $\varphi^{i}$ is a small oscillation around the equilibrium value $\varphi_{0}^{i}$ defined by $\varphi_{0}^{i}$ $=\sin ^{-1} \gamma^{i}$, we can then expand the $\sin \varphi^{i}$ term in $J^{i}$, and obtain the linerarized form of the full system, Eq. (1):

$$
\mathbf{S a}=\frac{k^{2}}{\omega^{2}-\sqrt{1-\gamma^{2}}} \mathbf{a} \equiv \lambda_{m} \mathbf{a},
$$

where we have used the fact that $\left(\gamma^{i}\right)^{2}=\left(\gamma^{j}\right)^{2} \equiv \gamma^{2}$. The expression $\sqrt{1-\gamma^{2}}$ is called the (square of the) bias-dependent plasma frequency, which is usually denoted by $\omega_{p}$, i.e., $\omega_{p}^{2}$ $=\sqrt{1-\gamma^{2}}$.

Equation (7) is just a normal eigenvalue equation, and we can find both the eigenvalues $\lambda_{m}$ and the eigenvectors $\mathbf{a}_{m}$. They are given by ${ }^{18}$

$$
\begin{gathered}
\mathbf{a}_{m}^{i}=\sqrt{\frac{2}{N+1}} \sin \left(\frac{i(N-m+1) \pi}{N+1}\right), \\
\lambda_{m}=1-2 S \cos \left(\frac{m \pi}{N+1}\right), \quad m=1, \ldots, N .
\end{gathered}
$$

Using Eqs. (7) and (8), we get $N$ dispersion relations

$$
\omega^{2}=\sqrt{1-\gamma^{2}}+c_{m}^{2} k^{2},
$$

where $c_{m}$ is the group velocity of the mode, defined as

$$
c_{m} \equiv \lim _{k \rightarrow \infty} \frac{d \omega}{d k}=\frac{1}{\sqrt{1-2 S \cos \left(\frac{m \pi}{N+1}\right)}} .
$$

We have now found that there are $N$ different plasma modes in the $N$ stacked Josephson junctions, each satisfying a different dispersion relation and each moving with a different velocity. We also know the exact form of the plasma oscillations, which is trivial to write down. We will do this anyway, because we shall use it later:

$$
A_{m}^{i}(x, t)=\sqrt{\frac{2}{N+1}} \sin \left(\frac{i(N-m+1) \pi}{N+1}\right) \cos (k x-\omega t) .
$$

\section{BUNCHED MODE OF THE 3 JUNCTION STACK}

Before proceeding to the fluxon modes of the coupled long Josephson junction we will look into the mechanisms of bunching by considering the simplest nontrivial case, $N$ $=3$. This in-phase mode is particularly interesting for electronics applications such as generation of microwave power. ${ }^{20}$ We will in the following take the bias currents to be equal, i.e., $\gamma^{1}=\gamma^{2}=\gamma^{3}$.

Let us first write down the equations explicitly:

$$
\begin{gathered}
J^{1}=\frac{1}{1-2 S^{2}}\left[\varphi_{x x}^{1}-S \varphi_{x x}^{2}+S^{2}\left(\varphi_{x x}^{3}-\varphi_{x x}^{1}\right)\right], \\
J^{2}=\frac{1}{1-2 S^{2}}\left[\varphi_{x x}^{2}-S\left(\varphi_{x x}^{1}+\varphi_{x x}^{3}\right)\right], \\
J^{3}=\frac{1}{1-2 S^{2}}\left[\varphi_{x x}^{3}-S \varphi_{x x}^{2}+S^{2}\left(\varphi_{x x}^{1}-\varphi_{x x}^{3}\right)\right] .
\end{gathered}
$$

It is easy to see from the symmetry of the equations that a solution with $\varphi^{1}=\varphi^{3}$ is possible, so we will try to find such a solution. If the mode is bunched, there is not much differ- 
ence between layers 1 and 2 either, which leads us to the following ansatz on the phases:

$$
\begin{gathered}
\varphi^{1}=\varphi+\delta \varphi=\varphi^{3}, \\
\varphi^{2}=\varphi-\kappa \delta \varphi,
\end{gathered}
$$

where $\kappa$ is some, yet, unknown constant and $\delta \varphi$ represents a (small) difference between the solutions. Introducing this into Eqs. (12) yields the following equations:

$$
\begin{aligned}
& J^{1}=\frac{1}{1-2 S^{2}}\left[(1-S) \varphi_{x x}+(1+\kappa S) \delta \varphi_{x x}\right], \\
& J^{2}=\frac{1}{1-2 S^{2}}\left[(1-2 S) \varphi_{x x}-(\kappa+2 S) \delta \varphi_{x x}\right] .
\end{aligned}
$$

Now by using that $\sin (\varphi+\delta \varphi) \approx \sin \varphi+\delta \varphi \cos \varphi$ and calculating the difference $J^{1}-J^{2}$, we get the following equation:

$$
\begin{aligned}
\delta \varphi_{t t}+ & \alpha \delta \varphi_{t}+\cos (\varphi) \delta \varphi \\
= & \frac{1}{(1+\kappa)\left(1-2 S^{2}\right)} \\
& \times\left[S \varphi_{x x}+(1+2 S+(1+S) \kappa) \delta \varphi_{x x}\right] .
\end{aligned}
$$

This equation is coupled to an equation for $\varphi$, and a general solution of these two coupled equations will probably be quite complicated. But we may look at the solution where $\sin \varphi=\varphi_{x x}=0$. If the solution for $\varphi$ is some form of a $2 \pi$ kink, then this corresponds to looking at solutions away from the center, located at $x=v t+x_{0}$. Doing this, we end up with the following differential equation for $\delta \varphi$ :

$$
\delta \varphi_{\xi \xi}-\frac{1}{v^{2}-\frac{1+2 S+(1+S) \kappa}{(1+\kappa)\left(1-2 S^{2}\right)}}\left(\alpha v \delta \varphi_{\xi}-\delta \varphi\right)=0,
$$

where we have introduced the self-coordinate $\xi$ defined by $\xi=x-v t-x_{0}$. If we restrict ourselves to the interval $\xi<0$, the solution of this type of equation has an exponential decaying term and an exponential growing term. The exponential growing term contradicts our assumption that $\delta \varphi$ is small and is discarded. The nature of the remaining term depends on the magnitude of $v^{2}$. Thus, if

$$
v^{2}>\frac{1+2 S+(1+S) \kappa}{(1+\kappa)\left(1-2 S^{2}\right)},
$$

we get an oscillating solution (discarding terms of second order and higher in $\alpha$ ). From Ref. 21 we know that we get oscillations (Cherenkov radiation) above the lower characteristic velocity, $c^{-}=1 / \sqrt{1-\sqrt{2} S}$, so by equating the left-hard side and the right-hand side in Eq. (17) and inserting $v$ $=c^{-}$, we can determine $\kappa$, and we find $\kappa=\sqrt{2}$. It is interesting to note that we also get $\sqrt{2}$ when we take the ratio of the amplitudes for the in-phase mode of the plasma oscillations in layers 1 and 2, i.e., $A_{3}^{2} / A_{3}^{1}=\sqrt{2}$. Thus the relative amplitudes of oscillating parts of the fluxon solution are car-

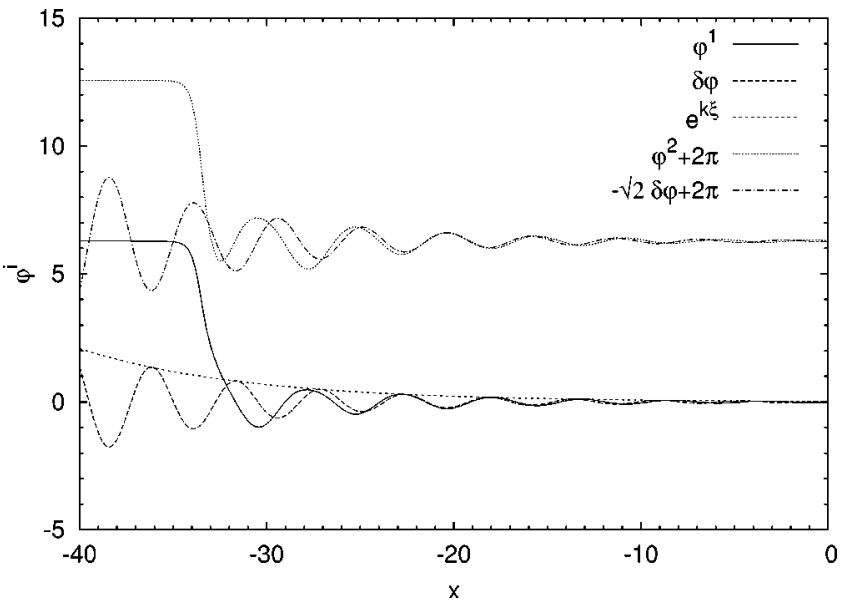

FIG. 2. Plot showing a numerical solution of the bunched mode of the 3 junction stack which clearly shows the oscillating tail. Also plotted is the analytical expression, Eq. (18), and the exponential decay curve is shown separately. The constants $A_{1}$ and $A_{2}$ have been fitted numerically. Parameters in the numerical solution are $\alpha=0.1, \gamma=-0.48, v=1.13$, and $S=-0.2$. $\varphi^{3}$ is not shown, because it is indistinguishable from $\varphi^{1}$. We see that the analytical expression fits well away from the fluxon center, which is expected from the assumptions made in the derivation of Eq. (18).

ried over from the plasma oscillations, but the phase oscillations are antiphase to lock the fluxons. ${ }^{22}$

Summarizing the characteristics of the bunched state, the amplitude in layer 2 is about $\sqrt{2}$ times bigger than the amplitude in layers 1 and 3, and the oscillations in the two layers are antiphase and exponentially decaying with a decay constant $k$ given by $k=\alpha v / 2\left(v^{2}-c_{-}^{2}\right)$. Finally, the angular frequency $\omega$ of the oscillations is given by $\omega=1 / \sqrt{v^{2}-c_{-}^{2}}$. Explicitly, we have for $\xi<0$ and $v>c^{-}$,

$$
\delta \varphi=e^{k \xi}\left(A_{1} \cos (\omega \xi)+A_{2} \sin (\omega \xi)\right)
$$

where $A_{1}$ and $A_{2}$ are some unknown constants. A plot showing the above formula together with a numerical solution of the full system can be seen in Fig. 2.

It is also worth noting that almost the same argument applies to the 2 junction stack (except we get $\kappa=1$ ). But here it does not represent the bunched state, because in the bunched state of the 2 junction stack, which corresponds to the ansatz (13), the phases are exactly equal, i.e., $\varphi^{1}=\varphi^{2}$. So the method in that case rather shows what happens when we have the bunched state, and then introduces some small difference between the two layers. The system responds with antiphase oscillations trying to preserve fluxon locking, for example, if $\varphi_{2}$ were trapped in a local minimum of $\varphi_{1}$. Several authors have investigated this scenario, see, for example, Ref. 22.

The role of the oscillations discussed for $N=3$ (and partly for $N=2$ ) are generic for the locking mechanism leading to in-phase fluxon motion. We note that the method should be usable for the cases $N>3$ also, but for the lack of space, we do not discuss this here. Nevertheless, the in-phase fluxon motion for $N=3$ shows the close correspondence between the fluxon modes and the corresponding plasma modes that can be obtained analytically. 

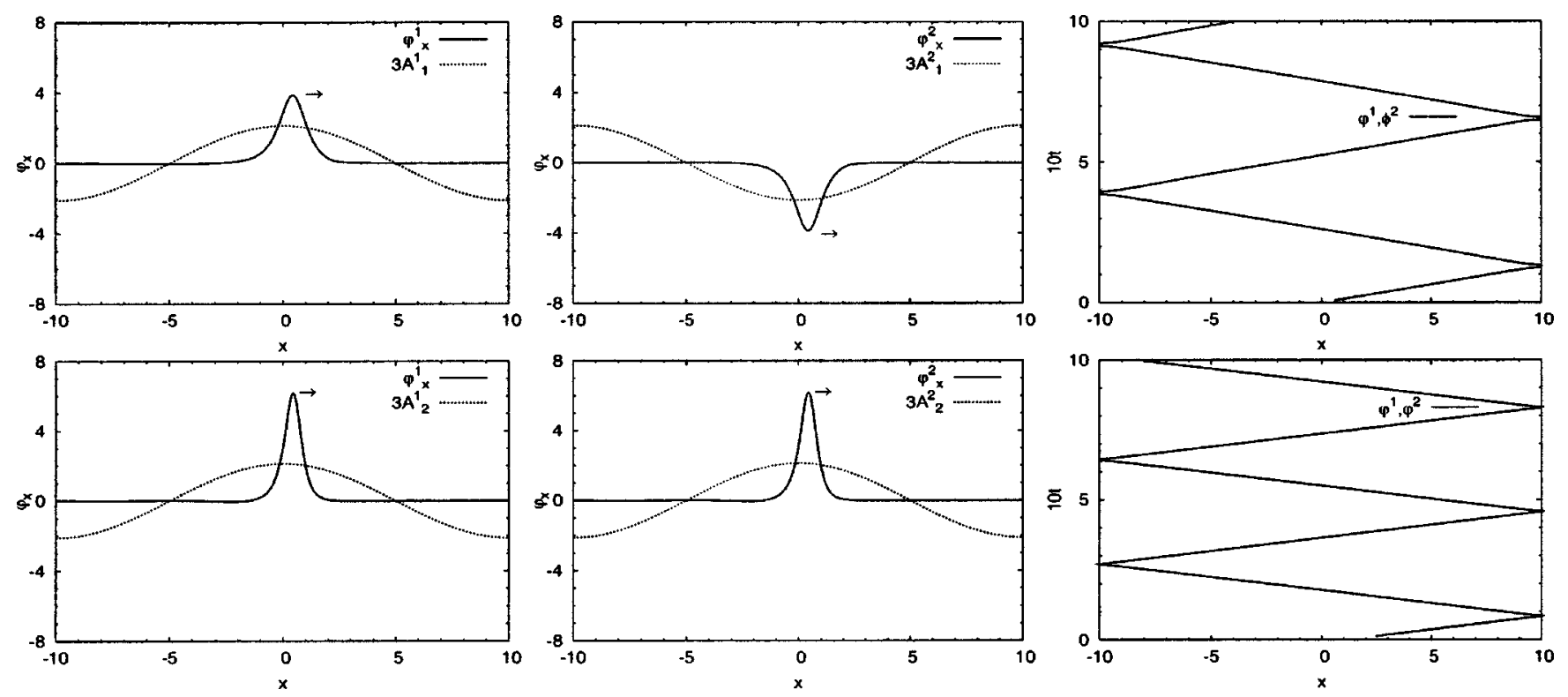

FIG. 3. Illustration of the could-be relationship between the plasma oscillations and the soliton modes of the 2 junction stack with unnatural biasing. The top plots are mode $1\left(\gamma^{1}=-\gamma^{2}\right)$ and the bottom plots are mode $2\left(\gamma^{1}=\gamma^{2}\right)$. The rightmost figures are the trajectories of the fluxons. Parameters are $\alpha=0.1, S=-0.2, \gamma=-0.2$ (top), -0.44 (bottom), and $v=0.75$ (top), 1.07 (bottom).

We also note from Eq. (11) for the $N=3$ system (with the bias currents we have chosen in this section) that in addition to the in-phase plasma oscillations there are two other plasma modes. Both of these are characterized by antiphase plasma oscillations. As we will discuss in the following section, this symmetry will suggest antiphase fluxon modes with little interaction and no antiphase oscillating tails. This will be demonstrated numerically in the following section.

\section{NUMERICAL SIMULATIONS}

In this section we will look numerically at a relationship between the plasma modes and the fluxon modes. We will first consider what we shall call "unnatural biasing." This is the case where $\gamma^{i}$ can be $\pm \gamma^{j}$. We refer to this as "unnatural," because it is hard, if not impossible, to realize this in an experimental setup for $N>2$. For $N=2$ it has been done experimentally, see Ref. 23. After this, we will focus on "natural biasing" $\left(\gamma^{i}=\gamma^{j}\right)$ and go into considerably more detail, because this is of greater practical interest. The results presented in this section should be regarded as "experimental" numerical observations and not as exact results.

For the 2 junction stack, there are two plasma modes: (a) the antiphase oscillations and (b) the in-phase oscillations. Following Ref. 23, there are, however, more fluxon modes than two. If the bias current has the same direction in both junctions, then there are (i) an in-phase fluxon-fluxon mode with characteristic velocity $c^{+}$and (ii) an antiphase fluxonantifluxon mode with characteristic velocity $c^{-}$. If the bias current has opposite directions in the two junctions (see Refs. 23 and 19), there are in addition (iii) an in-phase fluxon-antifluxon mode with characteristic velocity $c^{-}$and (iv) an antiphase fluxon-fluxon mode with characteristic velocity $c^{+}$. The latter is not discussed, but (i)-(iii) are shown in Figs. 3 and 4.

We will now investigate two types of mappings of the two plasma modes into the four fluxon modes. First, we choose the bias current as it suits our needs and next we change to the natural way of choosing the bias current. We will in the two following sections also see what happens when the mapping is generalized to $N>2$.

\section{A. Unnatural biasing}

For the 2 junction stack, we can map the two plasma modes into two in-phase fluxon modes. We thus state that the antiphase plasma oscillations map into the in-phase fluxonantifluxon mode (iii) and the in-phase plasma oscillations map into the in-phase fluxon-fluxon mode (i). This requires us to choose the bias current in different ways for the two modes, i.e., in opposite directions for (iii) and in same directions for (i). We will expect the velocities of the two modes to be smaller than the characteristic velocity for each mode. We know this is true from Ref. 23. The mapping is illustrated in Fig. 3. Note that the plasma oscillations in this and all other plots have been multiplied by 3 in order to show them more clearly.

The above illustrates the mapping for the 2 junction stack. We will generalize this, so that a mode consists of at most one fluxon (or antifluxon) in each junction, and the polarity of the fluxons is determined by the relative phases of the plasma oscillations. To be more precise, we use the following scheme to generate the fluxon/antifluxon configuration in mode $m:{ }^{29}$ (a) $a_{m}^{i}>0$, junction $i$ contains a fluxon; (b) $a_{m}^{i}$ $<0$, junction $i$ contains an antifluxon; (c) $a_{m}^{i}=0$, junction $i$ has no $2 \pi$ phase shift, and the velocity of the fluxons of mode $m$ should be in the interval $\left[0, c_{m}\right]$, because $c_{m}$ is the maximum velocity of the mode.

Next, we choose the bias current such that it drives all the fluxons and antifluxons in the same direction. One may say that we "fit" the bias current to suit our need for in-phase fluxon/antifluxon motion. The careful reader will here notice 

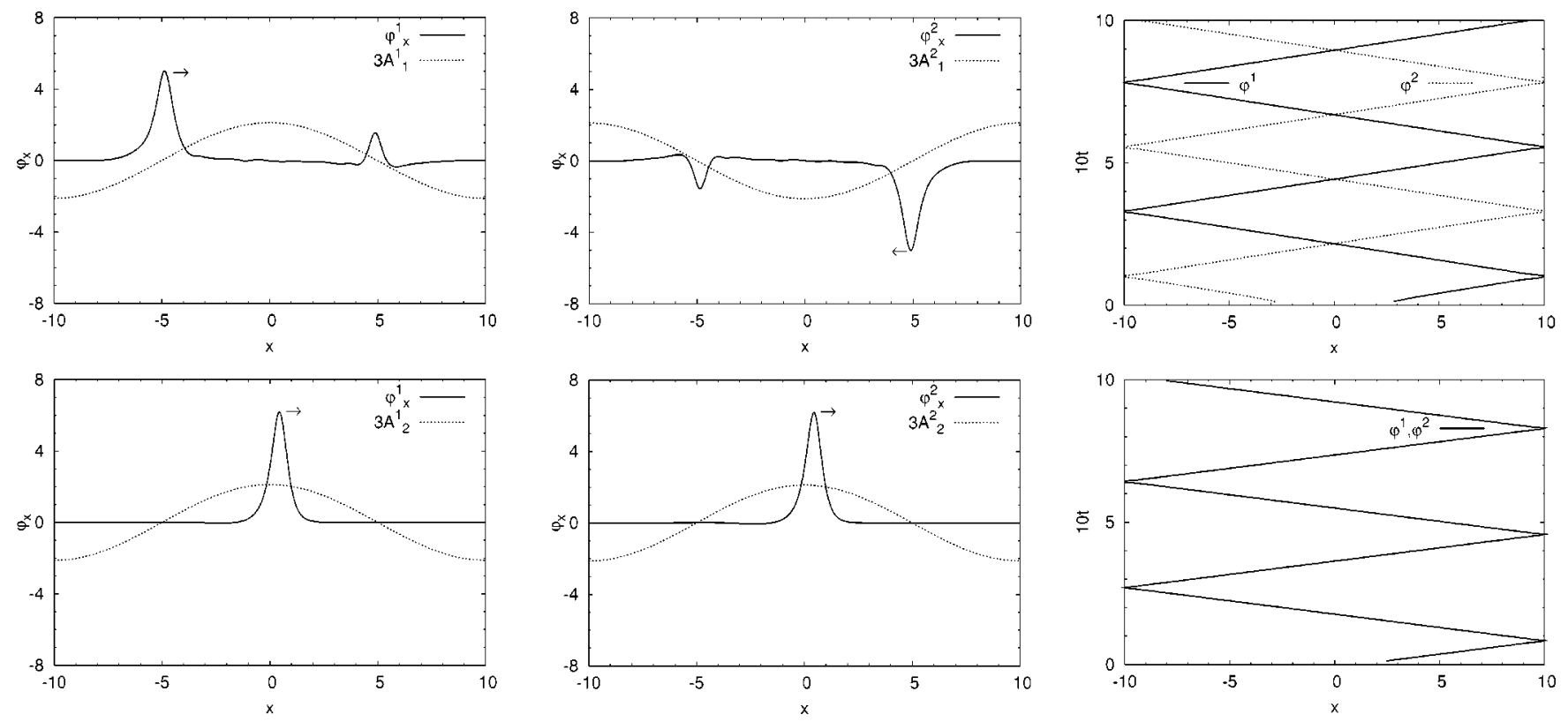

FIG. 4. Illustration of the could-be relationship between the plasma oscillations and the soliton modes of the 2 junction stack with natural biasing. The top plots are mode 1 and the bottom plots are mode 2. The rightmost figures are the trajectories of the fluxons. Parameters are $\alpha=0.1, S=-0.2, \gamma=-0.3$ (top), -0.44 (bottom), and $v=0.87$ (top), 1.07 (bottom).

that for the highest mode $(m=N)$, where all solitons have the same polarity, this unnatural way of choosing the bias current actually becomes the natural way. We will, nevertheless, refer to this way of choosing the bias current as unnatural, although it is only unnatural in $N-1$ modes out of a total of $N$ modes.

As we have already seen, these kinds of modes are possible in the 2 junction stack. We have carried this further, and found solutions according to the above construction of a mode for a stack with up to seven junctions.

To search for a given mode, we have used a standard procedure to solve the coupled non-linear equations (1). The equations have been discretized in the $x$ direction by a symmetric second-order finite-difference method, and the (large number of) resulting coupled ordinary differential equations have then been solved with a fifth-order Runge-Kutta method with error estimation valid to fourth order. The boundary conditions have been taken to be of the reflective type, Eq. (5).

In order to find the modes, we constructed an initial fluxon configuration using the above scheme. We choose some values of $\alpha,|\gamma|, L$, and $S$ and observed how the configuration evolved in time. If we did not find a satisfactory steady-state solution, we then tried to change the bias current $|\gamma|$, while holding $\alpha, L$, and $S$ fixed. We started with the lowest-order mode, and a low value of $|\gamma|$. From known $I V$ curves of small stacks we know that the modes will probably only exist for some finite range of $|\gamma|$, and that the lowest value of $|\gamma|$ where a mode is stable is increasing with the mode number. ${ }^{13}$ This is why we begin searching for the lowest mode to get an idea of what $|\gamma|$ we can use to find the mode. Also, the soliton velocity is increasing with the mode number, so it is also fair to assume that we must increase the bias current to achieve this.
We have been able to find all the modes, but with some minor discrepancies, which we will now point out. For stack sizes of more than three junctions, we have observed that the modes with the highest mode number $m=N$, the outer junctions are switching to the McCumber curve (finite voltage), but everything else is as expected. We will comment more on these modes in the section with natural biasing. In addition to this, we have found four other modes, which also only showed the expected behavior if some of the junctions had switched to finite voltage. We observed the following, in addition to the switched $m=N$ modes.

\begin{tabular}{cccc}
$N$ & $m$ & Expected & Found \\
\hline 4 & 3 & $\uparrow \uparrow \downarrow \downarrow$ & $\uparrow M M \downarrow$ \\
6 & 4 & $\uparrow \uparrow \downarrow \downarrow \uparrow \uparrow$ & $\uparrow M \downarrow \downarrow M \uparrow$ \\
6 & 5 & $\uparrow \uparrow \uparrow \downarrow \downarrow \downarrow$ & $\uparrow \uparrow M M \downarrow \downarrow$ \\
7 & 5 & $\uparrow \uparrow \downarrow \downarrow \downarrow \uparrow \uparrow$ & $\uparrow \uparrow M \downarrow M \uparrow \uparrow$ \\
\hline
\end{tabular}

where $\uparrow$ means $a_{m}^{i}>0, \downarrow$ means $a_{m}^{i}<0$, and $M$ means that the junction has switched to the McCumber curve. Junction number 1 is the leftmost arrow in each row, and the junction number is increasing as we move to the right. The results were calculated with $S=-0.2$ and $\alpha=0.1$, but we have also tried other values, and believe it to be valid for a wide range of parameters.

We thus see that the scheme predicts, rather reliably, a number of different fluxon configurations.

\section{B. Natural biasing}

For the case of natural biasing, it was recently proposed in Ref. 24 that there also, in this case, could exist a relationship between the linear modes and the fluxon modes of a Joseph- 

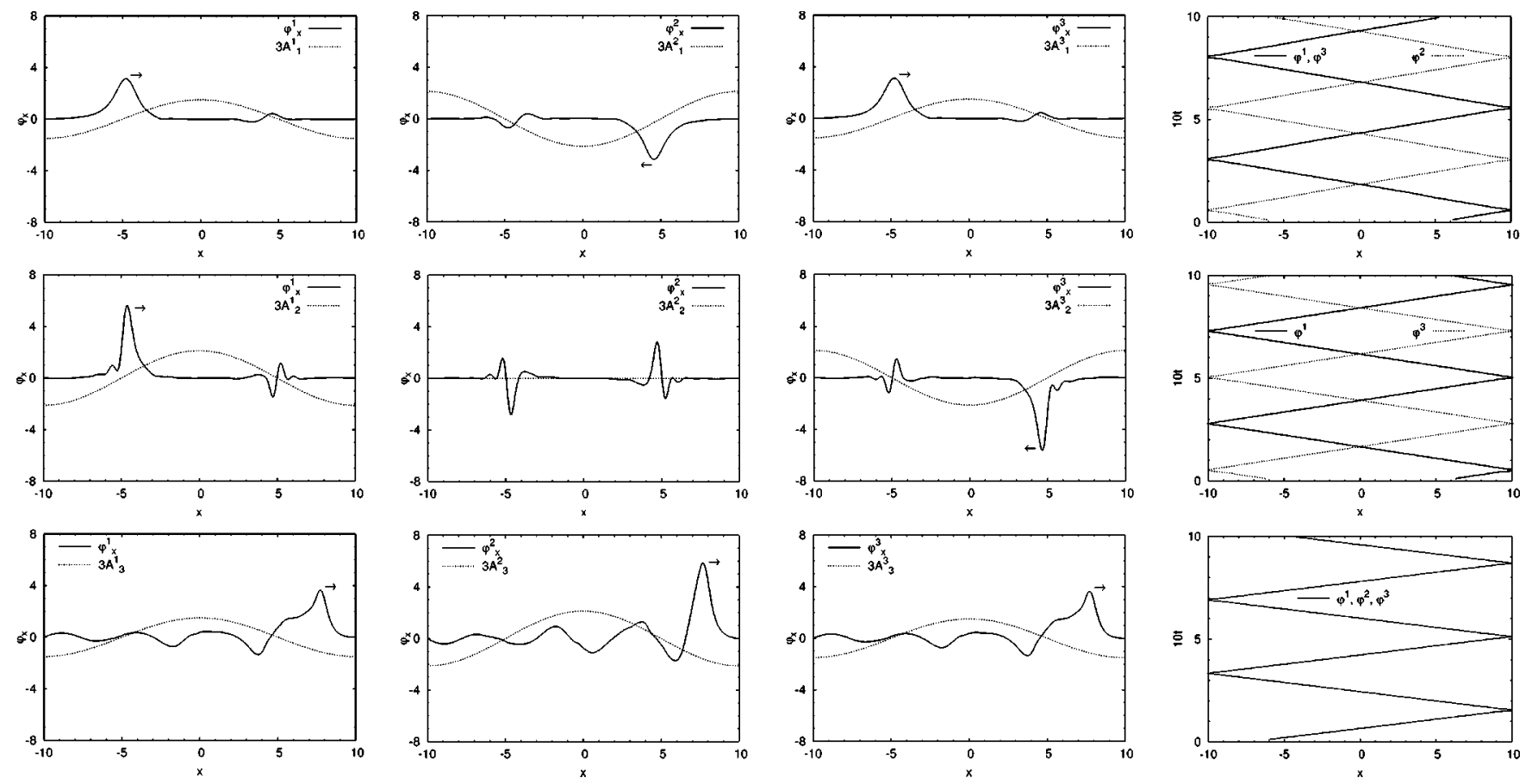

FIG. 5. The plasma oscillations and the soliton modes of the 3 junction stack with natural biasing. We see that the scheme also carries through for the 3 junction stack. The middle parts have no fluxon in the middle layer, thus only the first and second layers are present in the trajectories plot. Parameters for the figures are $\alpha=0.1, S=-0.2, \gamma=-0.2$ (top), -0.375 (middle), -0.44 (bottom), and $v=0.79$ (top), 0.89 (middle), 1.12 (bottom).

son stack. For a 2 junction stack it was noted that the antiphase plasma oscillations with characteristic velocity $c^{-}$ could correspond to the mode (ii) which has an antiphase fluxon motion, and the in-phase plasma oscillations with characteristic velocity $c^{+}$could correspond to the mode with in-phase fluxon motion (i). The similarity of the plasma oscillations and the soliton mode is illustrated in Fig. 4 for the 2 junction stack. For the antiphase mode in Fig. 4, the smaller peak is not a fluxon but the trace of the fluxon in the other junctions. This was also observed in Ref. 9.

We note that this behavior is expected if we assume the relationship proposed in Sec. V A. The relative polarities of the fluxons are the same as in Sec. V A, so the only difference is the bias current. If the bias current is chosen to be the same in all junctions, then fluxons and antifluxons will be driven in different directions. It is therefore expected that for a 2 junction stack mode $m=1$ will translate into fluxons moving in an antiphase pattern, i.e., we get the situation (ii) discussed in the beginning of this section.

We will in this section try to elaborate on the proposal from Ref. 24 by searching for similar modes in stacks with more than two junctions. The procedure is simply to see if there is a soliton mode which corresponds to a given plasma mode. The idea is to generalize the observation by Ref. 24 of the 2 junction stack. To be more precise, there should exist a steady-state soliton configuration in mode $m$, where the relative polarities are chosen according to the scheme in Sec. V A. We should expect that solitons should move in an antiphase manner with antisolitons, since the bias current drives them in opposite directions. Finally, the velocity $v$ of the fluxons should a priori belong to the interval $\left[0, c_{m}\right]$. We do not expect the upper bound to be exactly valid, because it is known even for the one-junction case that, when examined carefully, it does not hold exactly.

We have already seen in Fig. 4 that the 2 junction stack behaves just as proposed. In Fig. 4 we have also included a plot of the trajectories of the fluxons. These are found numerically by determining the position of a fluxon in layer $i$ by finding the lowest value of $\varphi_{t}^{i}$. In order to get the value as good as possible, without too large a number of $x$-mesh points, we had to approximate the shape of $\varphi_{t}$ around the minimum by a second-order polynomiad. Doing this at several times and plotting the positions as a function of time, we end up with the rightmost plot in Fig. 4. It is easy to see the fluxon motion of the two modes in this type of plot.

After being encouraged by the 2 junction stack we, naturally, analyzed the 3 junction stack. The 3 junction stack has been investigated by several groups, ${ }^{22,25-27}$ since it represents the lowest-order nontrivial system. For the expected in-phase fluxon mode, the fluxon-plasma relations were discussed perturbatively in the preceding section.

The results from the 3 junction stack is presented in Fig. 5. This figure shows that the lowest mode (top) has the plasma oscillations of layers 1 and 3 in-phase and layer 2 antiphase with layers 1 and 3 . We have then found a soliton mode where layers 1 and 3 are moving together and layer 2 is moving in an antiphase manner with these two layers. The magnitude of the velocity of the solitons is the same, namely; $v=0.79$ for the parameters used in the figure. This velocity is indeed in the range $\left[0, c_{1}\right]$ where, from Eq. (10), $c_{1} \approx 0.88$ when $S=-0.2$. 

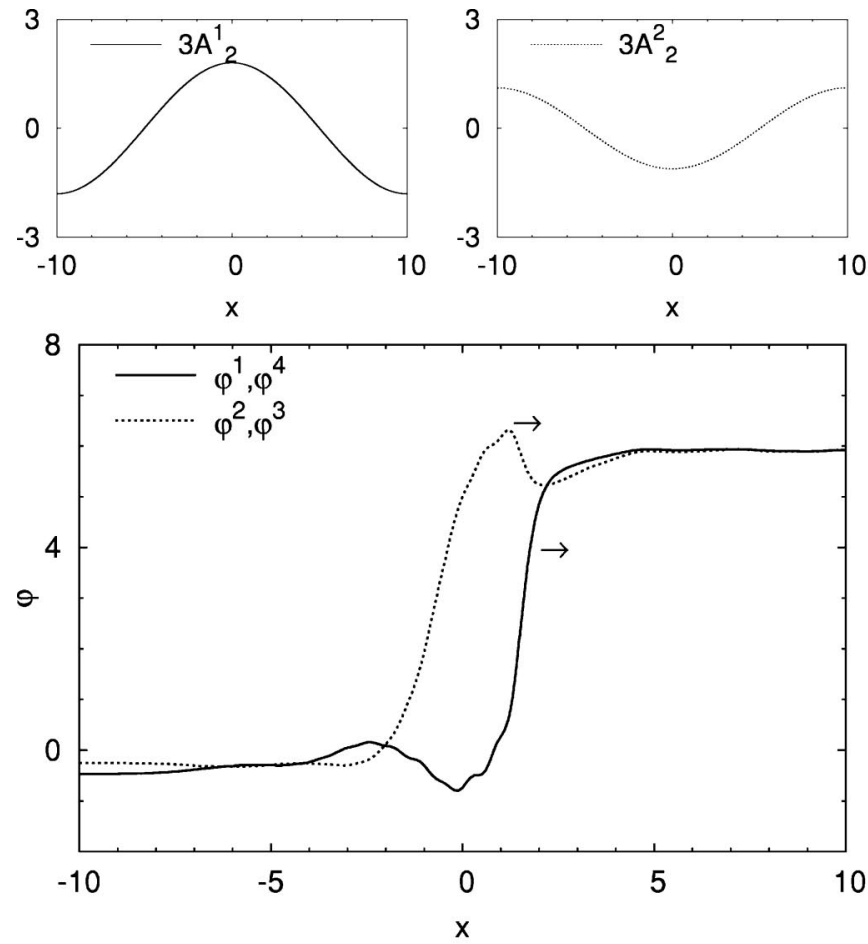
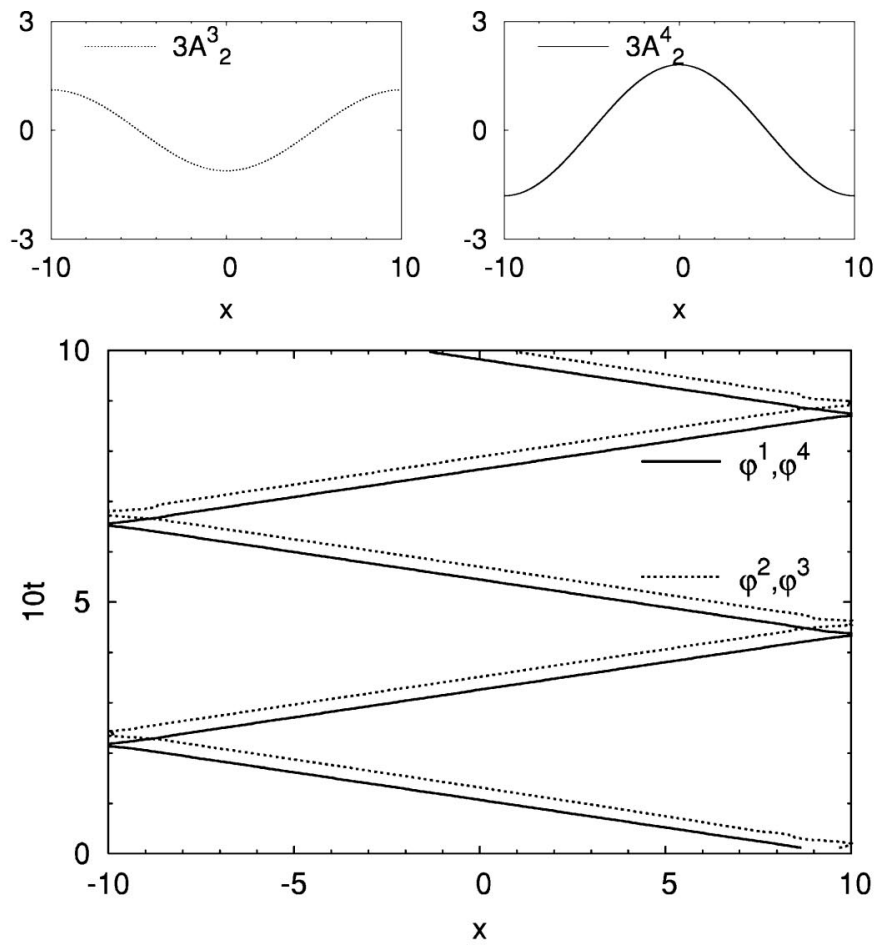

FIG. 6. Top plots are the plasmaoscillations of the second mode of the 4 junction stack with natural biasing. The bottom-left plot is for the phases $\phi^{i}$ of this mode and the bottom-right is for the trajectories of the solitons. Parameters for the plots are $\alpha=0.1, \gamma=-0.35, S$ $=-0.2$, and the velocity is $v=0.92$.

Although the present paper is considered as an empirical observation of a plasma mode-fluxon mode relationship, we note that a careful calculation of the trajectory in the top right of Fig. 5 shows that the $\varphi^{2}$ velocity is slightly different from the $\varphi^{1}$ and $\varphi^{3}$ velocity, and may even exceed the $c_{1}$ velocity. Such subtleties (mostly not visible to the naked eye) are sometimes observed, but beyond the empirical scope of the present paper.

The second mode of the 3 junction stack is seen as the middle plots of Fig. 5. Here the plasma oscillations tell us that we should look for a soliton mode where layers 1 and 3 are moving in antiphase and layer 2 contains no soliton. This is indeed found, and we get the magnitude of the velocities of the solitons in layers 1 and 3 to be $v=0.89$, which is well below $c_{2}$ when $S=-0.2$, and therefore in the desired range.

The third mode predicted by the proposal was also found, and it is the well known and very desirable in-phase mode of the 3 junction stack, which was also discussed in Sec. IV. It is shown in the bottom plots of Fig. 5. We again find that the mode conforms to the proposal, and that the velocity is in the desired range.

A very interesting question is now how well the proposal predicts the soliton modes when there are more than three junctions. We have found that it can predict most of the fluxon modes, but not all. We will here just explain the ones which deviate from the proposed behavior. It must be noted, though, that in the nonpredicted modes the basic symmetries of the plasma modes are still preserved.

For the 4 junction stack we find that the second mode deviates from what is expected. From the plasma oscillations we would expect that layers 1 and 4 should bunch and so should layers 2 and 3. This is also true, but we would also expect that layers 1 and 4 are antiphase with respect to layers 2 and 3, which is not what we observed. Instead, we find that all the layers are, more or less, bunched. Layers 1 and 4 are moving together very closely and so are layers 2 and 3, which are also moving a little behind layers 1 and 4 . All solitons are moving with the same velocity, which is in the desired interval $\left[0, c_{2}\right]$. The mode can be seen in Fig. 6 .

We have also found that the fourth mode of the 4 junction stack deviates from the expected behavior, which was also noted in Sec. V A. This is the mode which should be totally bunched, but we find that the solitons in the two outer layers have disappeared, and instead the layers have switched to the McCumber curve $\left(\varphi_{t} \sim \gamma / \alpha\right)$. Although there is no soliton in the outer layers, we still have an image in the outer layers from the solitons in the middle layers. This means that $\varphi_{x}^{i}$ looks more or less like there is a soliton in all the layers, as can be seen in Fig. 7. Interest in this mode is usually due to the fact that it can be used as a microwave oscillator, where we get pulses at the edges when the solitons arrive. These pulses depend on the voltage across the junction, which is proportional to $\varphi_{t}$. Judging from Fig. 7, it should not matter that much if the outer layers have switched to the McCumber curve, because the pulsed microwave output is of the same order. For cases similar to Fig. 7 we have confirmed from numerical simulations of the available total power from all the junctions that this is indeed the case. ${ }^{28}$

We have summarized our results in Table I. For up to a 7 junction stack we discuss in Table I the validity of our initial proposal that it is possible to predict a family of fluxon 

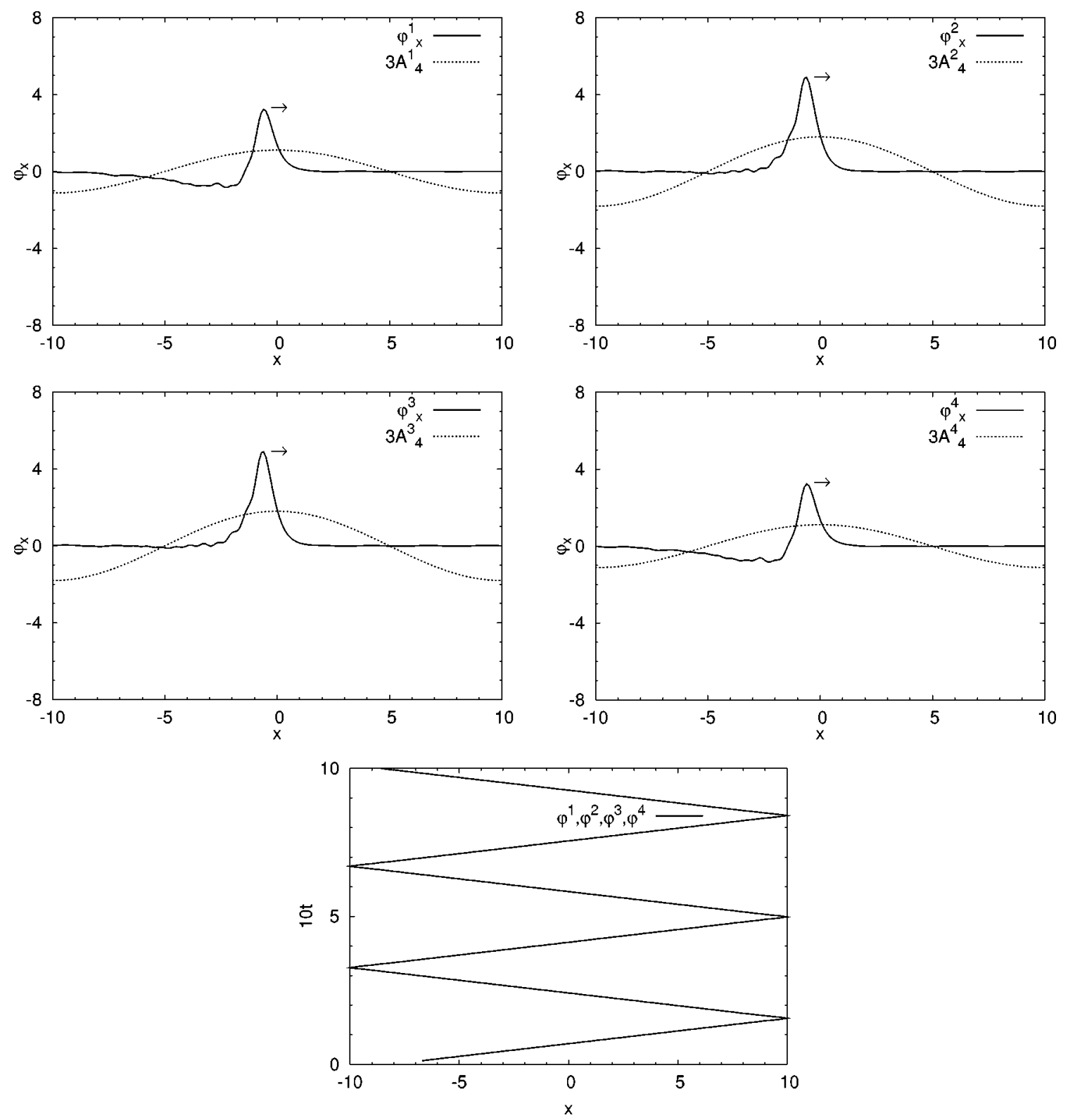

FIG. 7. The top four plots are $\phi_{x}$ of the fourth mode of the 4 junction stack with natural biasing together with the plasma oscillations of this mode. We see that it is hard to tell that there is no soliton present in the first and fourth layers from $\phi_{x}$. The bottom plot is the trajectories of the "solitons." Parameters for the plots are $\alpha=0.1, \gamma=-0.54, S=-0.2$, and the velocity is $v=1.17$.

modes by just looking at the analytical expression of the plasma modes. We note that we use a different short-hand notation than in the preceding section. We do this to emphasize the fluxon motion and not the fluxon polarity.

In this generic prediction scheme, an upward pointing plasma excitation leads to a fluxon, a downward pointing plasma excitation leads to an antifluxon, and zero-amplitude plasma excitation leads to no fluxon.

In Table I the horizontal direction gives the number of junctions in the stack, $N$, and the vertical direction gives the order of the mode, with mode 1 always being the clean antiphase mode and mode $N$ being the in-phase mode (at least for the plasma excitation). In each square, the right-hand side shows the plasma-mode prediction and the left part shows the fluxon-mode obtained from numerical simulations. The trivial case $N=1$, which has both a plasma mode and a fluxon mode in agreement with the prediction, has been omitted in Table I.

Moving horizontally, we see in the first set of squares the predicted antiphase fluxon modes with every fluxon moving in opposite direction to that in the neighboring layer. If we follow the squares in the direction of the diagonal, the plasma-mode prediction method suggests in-phase fluxon modes. This is also found, except for higher $N$. Here the two outer layer fluxons are replaced by a similar looking pulse (in-phase with the other fluxons) but on top of the voltage corresponding to the McCumber curve. A different value of the layer-to-layer coupling $S$ could presumably convert the outer pulses back to clean fluxon pulses; however, as discussed above, the predicted pulse geometry is preserved, and 
TABLE I. Summary of what we have found with natural biasing. An arrow means a fluxon, a line means no fluxon, a dashed line means the layer is on the McCumber curve, a dashed arrow means McCumber with a clear image of a fluxon, $\times$ means that something is not according to the proposal, $M$ means that some layers have switched to the McCumber curve but otherwise okay, and $\checkmark$ means that everything is according to the proposal. The arrows to the left of the symbols, $\sqrt{ }, M$ and $\times$, show the mode we have found, and the ones to the right show the mode according to the proposal. A small horisontal shift between arrows implies that the fluxon distance (away from the boundaries) in $x$ space is greater than $\lambda_{J}$, and no spacing means that the distance is much smaller than $\lambda_{J}$.

\begin{tabular}{|c|c|c|c|c|c|c|c|c|c|c|c|c|}
\hline Stack Size & 2 & 3 & & 4 & & 5 & & t & & \multicolumn{3}{|c|}{7} \\
\hline Mode 1 & $\leftarrow \sqrt{ } \leftarrow$ & $\begin{array}{lll}\rightarrow & & \\
\leftarrow & \sqrt{ } & \\
\rightarrow & \end{array}$ & $\begin{array}{l}\rightarrow \\
\leftarrow \\
\rightarrow\end{array}$ & $\begin{array}{l}\leftarrow \\
\rightarrow \quad \\
\leftarrow \\
\rightarrow\end{array}$ & $\begin{array}{l}\leftarrow \\
\leftarrow \\
\leftarrow \\
\rightarrow\end{array}$ & $\begin{array}{l}\rightarrow \\
\leftarrow \quad \\
\leftarrow \\
\rightarrow\end{array}$ & $\begin{array}{l}\rightarrow \\
\leftarrow \\
\leftarrow \\
\rightarrow\end{array}$ & $\begin{array}{l}\leftarrow \\
\leftarrow \\
\leftarrow \\
\leftarrow \\
\leftarrow\end{array}$ & $\begin{array}{l}\leftarrow \\
\leftarrow \\
\leftarrow \\
\leftarrow \\
\leftarrow \\
\rightarrow\end{array}$ & $\begin{array}{l}\rightarrow \\
\leftarrow \\
\leftarrow \\
\rightarrow \\
\leftarrow \\
\rightarrow\end{array}$ & & $\begin{array}{l}\rightarrow \\
\leftarrow \\
\rightarrow \\
\leftarrow \\
\rightarrow \\
\leftarrow \\
\rightarrow\end{array}$ \\
\hline Mode 2 & $\rightarrow \quad \sqrt{ }$ & $\begin{array}{ll}\rightarrow & \\
- & \sqrt{ } \\
\leftarrow & \end{array}$ & $\begin{array}{l}\rightarrow \\
- \\
\leftarrow\end{array}$ & $\begin{array}{l}\rightarrow \\
\rightarrow \\
\rightarrow\end{array}$ & $\begin{array}{l}\rightarrow \\
\leftarrow \\
\leftarrow \\
\rightarrow\end{array}$ & $\begin{array}{l}\leftarrow \\
\rightarrow \\
-\quad \sqrt{ } \\
\leftarrow \\
\rightarrow\end{array}$ & $\begin{array}{l}\leftarrow \\
\rightarrow \\
- \\
\leftarrow \\
\rightarrow\end{array}$ & $\begin{array}{l}\rightarrow \\
\rightarrow \\
\rightarrow \\
\rightarrow \\
\rightarrow\end{array}$ & $\begin{array}{l}\rightarrow \\
\leftarrow \\
\rightarrow \\
\leftarrow \\
\rightarrow\end{array}$ & $\begin{array}{l}\leftarrow \\
\rightarrow \\
\leftarrow \\
- \\
\leftarrow \\
\leftarrow\end{array}$ & & $\begin{array}{l}\leftarrow \\
\leftarrow \\
- \\
\rightarrow \\
\leftarrow \\
\rightarrow\end{array}$ \\
\hline Mode 3 & & $\begin{array}{ll}\rightarrow & \\
\rightarrow & \sqrt{ } \\
\rightarrow & \end{array}$ & $\begin{array}{l}\rightarrow \\
\rightarrow\end{array}$ & $\begin{array}{l}\leftarrow \\
\leftarrow \\
\rightarrow\end{array}$ & $\begin{array}{l}\leftarrow \\
\leftarrow \\
\rightarrow \\
\rightarrow\end{array}$ & $\begin{array}{l}\rightarrow \\
- \\
\rightarrow \\
- \\
\rightarrow\end{array}$ & $\begin{array}{l}\rightarrow \\
- \\
\leftarrow \\
\rightarrow\end{array}$ & $\begin{array}{l}- \\
\rightarrow \\
\rightarrow \quad> \\
\leftarrow \\
\leftarrow \\
-\end{array}$ & $\begin{array}{l}\leftarrow \\
\rightarrow \\
\leftarrow \\
\leftarrow \\
\leftarrow \\
\rightarrow\end{array}$ & $\begin{array}{l}\rightarrow \\
- \\
- \\
\rightarrow \\
- \\
- \\
\rightarrow\end{array}$ & & $\begin{array}{l}\rightarrow \\
\leftarrow \\
\leftarrow \\
\rightarrow \\
\leftarrow \\
\leftarrow \\
\rightarrow\end{array}$ \\
\hline Mode 4 & & & & $\begin{array}{l}--\rightarrow \\
\rightarrow \\
\rightarrow\end{array}$ & $\begin{array}{l}\rightarrow \\
\rightarrow \\
\rightarrow\end{array}$ & $\begin{array}{l}\leftarrow \\
\leftarrow \quad \sqrt{ } \\
\rightarrow \\
\rightarrow\end{array}$ & $\begin{array}{l}\leftarrow \\
\leftarrow \\
- \\
\rightarrow\end{array}$ & $\begin{array}{l}\rightarrow \\
\rightarrow \\
- \\
- \\
\rightarrow\end{array}$ & $\begin{array}{l}\rightarrow \\
\rightarrow \\
\leftarrow \\
\leftarrow \\
\rightarrow\end{array}$ & $\begin{array}{l}\leftarrow \\
- \\
\rightarrow \\
- \\
\leftarrow \\
- \\
\rightarrow\end{array}$ & & $\begin{array}{l}\leftarrow \\
- \\
\rightarrow \\
- \\
\leftarrow \\
- \\
\rightarrow\end{array}$ \\
\hline
\end{tabular}


TABLE I. (Continued).

\begin{tabular}{|c|c|c|c|c|c|c|c|c|c|c|}
\hline Stack Size & 2 & 3 & 4 & & & & & \multicolumn{3}{|c|}{7} \\
\hline Mode 5 & & & & $\begin{array}{l}--\rightarrow \\
\rightarrow \\
\rightarrow \\
\rightarrow \\
-\rightarrow\end{array}$ & $\begin{array}{r}\rightarrow \\
\rightarrow \\
\rightarrow \\
\rightarrow \\
\rightarrow\end{array}$ & $\begin{array}{l}\leftarrow \\
\leftarrow \\
\leftarrow \\
\rightarrow \\
\rightarrow \\
\rightarrow\end{array}$ & $\begin{array}{l}\leftarrow \\
\leftarrow \\
\leftarrow \\
\rightarrow \\
\rightarrow\end{array}$ & $\begin{array}{l}\rightarrow \\
\rightarrow \\
\rightarrow \\
- \\
\rightarrow \\
\rightarrow\end{array}$ & 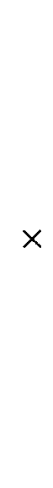 & $\begin{array}{l}\rightarrow \\
\rightarrow \\
\leftarrow \\
\leftarrow \\
\leftarrow \\
\rightarrow\end{array}$ \\
\hline Mode 6 & & & & & & $\begin{array}{l}-\rightarrow \\
\rightarrow \\
\rightarrow \\
\rightarrow \\
\rightarrow\end{array}$ & $\begin{array}{l}\rightarrow \\
\rightarrow \\
\rightarrow \\
\rightarrow \\
\rightarrow\end{array}$ & $\begin{array}{l}+ \\
\leftarrow \\
\leftarrow \\
+ \\
\rightarrow \\
\rightarrow \\
-\end{array}$ & 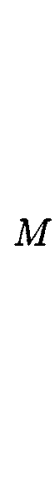 & $\begin{array}{l}\leftarrow \\
\leftarrow \\
\leftarrow \\
- \\
\rightarrow \\
\rightarrow\end{array}$ \\
\hline Mode 7 & & & & & & & & $\begin{array}{l}-- \\
\rightarrow \\
\rightarrow \\
\rightarrow \\
\rightarrow \\
\rightarrow \\
-\end{array}$ & $M$ & $\begin{array}{l}\rightarrow \\
\rightarrow \\
\rightarrow \\
\rightarrow \\
\rightarrow \\
\rightarrow\end{array}$ \\
\hline
\end{tabular}

the change is not essential for the total power output from the in-phase pulses, as we have confirmed in numerical simulations. $^{28}$

Even the squares with crosses, which indicate discrepancies, show that the numerically obtained fluxon mode has a majority of the features in common with the prediction from the linear plasma modes.

We note that the plasma modes and fluxon modes have not only their geometry in common, but also their velocity. For the plasma modes this velocity is easily obtained analytically, or can be taken from the dispersion relation. Finding the same approximate velocity in the fluxon modes just confirms the validity of our prediction.

We point out that our method should be applicable also for more complicated modes, for example, with more than one fluxon in each layer, or if the stack is subject to an in-plane magnetic field.

\section{CONCLUSION}

We have considered a stack of inductively coupled Josephson junctions with particular emphasis on the symmetries of the plasma modes, which can be derived analytically. We demonstrated empirically, by numerical methods, that the properties of the fluxon modes can be rather reliably predicted from the symmetries of the plasma modes. This allowed us to numerically find a family of soliton modes, using only knowledge from the plasma modes. Other authors noted that the plasma modes enter the soliton modes in the form of Cherenkov radiation. As a side result, we were able to calculate the decay profile of this radiation analytically. 


\section{ACKNOWLEDGMENTS}

We acknowledge the support from the ESF VORTEX program, the STVF framework program "New Superconductors: Mechanisms, processes and products" and NATO "Sci- ence for Peace." S.M. would also like to thank M. P. Sørensen for discussions and help regarding the numerics. We also thank A. Ustinov for critical reading of the manuscript and helpful comments.
*Electronic address: Soeren@Madsen2TheMax.dk

†Electronic address: nfp@oersted.dtu.dk

${ }^{1}$ Intrinsic Josephson Effects and Plasma Oscillations in High- $\mathrm{T}_{\mathrm{c}}$ Superconductors, edited by M. Tachiki and T. Yamashita (Elsevier, The Netherlands, 2001).

${ }^{2}$ T. Yamashita, S.J. Kim, Y. Latyshev, and K. Nakjima, Physica C 335, 219 (2000).

${ }^{3}$ Yu.I. Latyshev, A.E. Koshelev, and L.N. Bulaevskii (unpublished).

${ }^{4}$ S. Heim, M. Mößle, T. Clauß, and R. Kleiner, Supercond. Sci. Technol. 15, 1226 (2002).

${ }^{5}$ R. Kleiner, Phys. Rev. B 50, 6919 (1994).

${ }^{6}$ A.A. Yurgens, Supercond. Sci. Technol. 13, R85 (2000).

${ }^{7}$ K. Schlenga, R. Kleiner, G. Hechtfischer, M. Mößle, S. Schmitt, P. Müller, Ch. Helm, Ch. Preis, F. Forsthofer, J. Keller, H.L. Johnson, M. Veith, and E. Steinbeiß, Phys. Rev. B 57, 14518 (1998).

${ }^{8}$ H.B. Wang, Y. Aruga, T. Tachiki, Y. Mizugaki, J. Chen, and K. Nakajima, Appl. Phys. Lett. 74, 3693 (1999).

${ }^{9}$ S. Sakai, P. Bodin, and N.F. Pedersen, J. Appl. Phys. 73, 2411 (1993).

${ }^{10}$ N.F. Pedersen, in Solitons, edited by A.A. Maradudin and V.H. Agranowich (North-Holland, Amsterdam, 1986) p. 469.

${ }^{11}$ D.W. McLaughlin and A.C. Scott, Phys. Rev. A 18, 1652 (1978).

${ }^{12}$ Y.S. Kivshar and B.A. Malomed, Rev. Mod. Phys. 61, 763 (1989).

${ }^{13}$ A. Ustinov, Physica D 123, 315 (1998).

${ }^{14}$ J. Zitzmann, A.V. Ustinov, M. Levitchev, and S. Sakai, Phys. Rev. B 66, 064527 (2002).
${ }^{15}$ T. Clauß, V. Oehmichen, M. Mößle, A. Müller, A. Weber, D. Koelle, and R. Kleriner, Supercond. Sci. Technol. 15, 1651 (2002).

${ }^{16}$ S. Sakai and N.F. Pedersen, Phys. Rev. B 60, 9810 (1999).

${ }^{17}$ R. Kleiner, T. Gaber, and G. Hechtfischer, Phys. Rev. B 62, 4086 (2000).

${ }^{18}$ N.F. Pedersen and S. Sakai, Phys. Rev. B 58, 2820 (1998).

${ }^{19}$ A. Petraglia, A.V. Ustinov, N.F. Pedersen, and S. Sakai, J. Appl. Phys. 77, 1171 (1995).

${ }^{20}$ A.V. Ustinov and S. Sakai, Appl. Phys. Lett. 73, 868 (1998).

${ }^{21}$ E. Goldobin, A. Wallraff, N. Thyssen, and A.V. Ustinov, Phys. Rev. B 57, 130 (1998).

${ }^{22}$ E. Goldobin, B.A. Malomed, and A.V. Ustinov, Phys. Rev. B 62, 1414 (2000).

${ }^{23}$ G. Carapella, G. Costabile, A. Petraglia, N.F. Pedersen, and J. Mygind, Appl. Phys. Lett. 69, 1300 (1996).

${ }^{24}$ N.F. Pedersen and G. Filatrella, Physica C 369, 171 (2002).

${ }^{25}$ A.V. Ustinov, H. Kohlstedt, M. Cirillo, N.F. Pedersen, G. Hallmanns, and C. Heiden, Phys. Rev. B 48, 10614 (1993).

${ }^{26}$ C. Gorria, P.L. Christiansen, Yu.B. Gaididei, V. Muto, N.F. Pedersen, and M.P. Soerensen, Phys. Rev. B 68, 035415 (2003).

${ }^{27}$ C. Gorria, P.L. Christiansen, Yu.B. Gaididei, V. Muto, N.F. Pedersen, and M.P. Soerensen, Phys. Rev. B 66, 172503 (2002).

${ }^{28}$ N.F. Pedersen and S. Madsen (unpublished).

${ }^{29}$ The terms fluxon and antifluxons are of course arbitrary, because we use reflective boundary conditions. What we mean, which should be clear from the context, is that the relative polarities of the fluxons are determined by the signs of $a_{m}^{i}$. 